\title{
CEACAM1 is a Privileged Cell Surface Antigen to Design Novel ScFv Mediated-Immunotherapies of Melanoma, Lung Cancer and Other Types of Tumors
}

\author{
Maurizio Cianfriglia ${ }^{1}$, Valentina Fiori ${ }^{*}, 2$, Sabrina Dominici ${ }^{3}$, Silvia Zamboni ${ }^{1}$, Michela Flego ${ }^{1}$, \\ Maria Luisa Dupuis ${ }^{1}$, Alessandro Ascione ${ }^{1}$, Mara Gellini ${ }^{1}$, Alessandra Mallano ${ }^{1}$ and \\ Mauro Magnani ${ }^{3}$
}

${ }^{I}$ Section of Pharmacogenetics, Drug Resistance and Experimental Therapeutics. Department of Drug Research and Medicine Evaluation, Istituto Superiore di Sanità, Viale Regina Elena 299, 00161 Roma, Italy

${ }^{2}$ Diatheva srl, viale Piceno 137/F, 61032 Fano (PU), Italy

${ }^{3}$ Department of Biomolecular Sciences, University of Urbino 'Carlo Bo', Via Saffi 2, 61029 Urbino (PU), Italy

\begin{abstract}
Carcinoembryonic antigen-related cell adhesion molecule 1 (CEACAM1) is a cell surface glycoprotein involved in intercellular binding, belonging to the immunoglobulin superfamily. It is involved in cell-cell recognition and modulates cellular processes that range from vascular angiogenesis to the regulation of insulin homeostasis and T-cell proliferation. Aberrant expression of CEACAM1 is often associated with progression and metastatic potential in melanoma, lung carcinoma and other types of tumor. Tumor-specific antigens such as CEACAM1 are ideal targets for cancer immunotherapy because they are over-expressed by the cancer cell and not on non-malignant tissues, minimizing the risk of autoimmune destruction. Many of the limitations of therapeutic use of rodent monoclonal antibodies (mAbs) can now be overcome by exploiting the use of recombinant antibody fragments and the advances in antibody engineering methods to improve tumor retention, reduce immunogenicity and modulate pharmacokinetics. In addition, a novel effective model of immunotherapeutic treatments of tumors includes antibody drug conjugates (ADCs) that combine specific mAbs and antibody fragments with cytotoxic drugs, proteins, enzymes, radionuclides and nanoparticles. This review aims to describe how these antibody engineering approaches can meet the challenges for generating new and effective antibody constructs for diagnosis and therapy of CEACAM1 expressing malignancies.
\end{abstract}

Keywords: CEACAM1 expression, immunoconjugates, immunotheraphy, lung cancer, melanoma, phage display, scFv antibodies, tumor angiogenesis.

\section{INTRODUCTION}

Carcinoembryonic antigen-related cell adhesion molecules (CEACAMs) belong to a group of mammalian immunoglobulin-related glycoproteins [1]. They are implicated in various cellular functions governing growth and differentiation and have an important role in insulin homeostasis, vascular neogenesis, and on immune modulation [2-4]. Some CEACAMs members (CEACAM1, CEACAM3, CEACAM5 and CEACAM6) have also been identified as receptors for host-specific viruses and bacteria in mice and humans [5]. Further, CEACAMs have been implicated in various intracellular signalling-mediated effects involved in the growth and differentiation of cancerous cells thus playing a key role in the modulation of various type of cancers. The carcinoembryonic antigen (CEA; CEACAM5) represents one of the best-known tumorassociated antigens since it was isolated in 1965 from extracts of human colon cancers [6]. CEACAM5 is overexpressed in various tumors of epithelial origin of the

*Address correspondence to this author at the Diatheva srl, viale Piceno 137/F, 61032 Fano (PU), Italy. Phone: +39-0721830605; Fax: +390721837154; E-mail: v.fiori@diatheva.com digestive tract in particular those of the colon-rectum, stomach, pancreas, but also in other cancers (such as breast, lung, urinary tract, ovary and cervix) [7] and is routinely used as a marker in the diagnosis, staging, monitoring and prognosis of these tumors. Another member of the CEACAMs family, CEACAM1, has recently received considerable interest as a tumor antigen target especially in melanoma and lung cancer. CEACAM1 has the widest tissue distribution of all characterized family members. This receptor is found on different epithelial cells and its expression can be induced in endothelial or $\mathrm{T}$ cells [8]. CEACAM1 shows intercellular homophilic interactions as well as heterophilic binding to CEACAM5 expressed on target cells $[9,10]$. Clinical studies in a 10-year follow-up in melanomas show that expression of the cell adhesion molecule CEACAM1 is an independent factor of the metastasis risk, with a predictive value superior to that of tumor thickness [11]. Furthermore, CEACAM1, which is not expressed in the normal lung, has been identified in carcinomas from this tissue and correlates with poor survival [12]. In addition, a significant correlation was found between the high expression of CEACAM1 on cells of the primary tumor in pulmonary adenocarcinoma and metastasization [13]. CECAM1 also seems to be a promising endothelial 
target for the treatment of prostate cancer, this molecule is indeed involved in the transition from non-invasive and nonvascularized to invasive and vascularized cancer [14]. High levels of expression of CEACAM1 have been also associated with lymph node metastasis in gastric cancer [15] and tumor invasiveness in colorectal cancer $[16,17]$. Taken together, these findings raised the possibility that CEACAM1 expression might facilitate metastatic tumor spread. Since CEACAM1 exhibits properties of an angiogenic factor acting as a major effector of vascular endothelial growth factor (VEGF), it may promote metastasis by the induction of angiogenesis at the metastatic site $[3,18,19]$. In addition, in many tumors CEACAM1 expression is actively upregulated in response to interferon-gamma and homophilic CEACAM1 interaction may be directly involved in inhibiting natural killer and cytotoxic $\mathrm{T}$ cells effector functions. This acquired immune resistance could be responsible for the poor prognosis and enhanced resistance to cell mediated response observed in CEACAM1 overexpressing tumors [20-23].

Multi-modal treatments that combine chemotherapy with surgery or radiotherapy has increased the survival rate of tumor patients. In particular, dose-intensification has recently improved their cure rate, despite the increase of severe toxicity and high level of life-threatening late events, such as secondary malignancies [24]. The treatment of cancer remains a formidable challenge owing to the difficulties in differentiating tumor cells from healthy cells to ameliorate the disease without causing intolerable toxicity to patients. In addition, the emergence of MDR1-Pglycoprotein mediated multi-drug resistance (MDR) it is a not rare phenomenon that inhibits the curative potential of chemotherapeutic treatments [25]. One way to improve the selectivity of therapeutic molecules in tumors would be to target them on the tumor site, thereby sparing normal tissues. In this sense, the approaches based on monoclonal antibodies (mAbs) specific for tumor associated antigens represent a good strategy. The expression of CEACAM1 in the development of aggressive melanoma and several carcinomas marks this adhesion molecule as an attractive target for novel forms of monoclonal antibodies-based immunotherapeutic interventions. That of $\mathrm{mAbs}$ is the fastest growing sector of pharmaceutical biotechnology and a number of antibody-based biopharmaceuticals have been approved for cancer treatment $[26,27]$. However, in many cases the antibodies used for the treatment of solid tumors offer only a modest survival benefit to cancer patients thereby yielding marginal therapeutic indices. On the other hand, treatment in combination with small cytotoxic drugs significantly improves the clinical efficacy of naked antibody [28]. As a result, investigators have turned to arming mAbs with drugs, toxins or radionuclides.

In this review, we look into different approaches that have been followed during the past few years to produce antitumor therapeutics based on the utilization of single chain fragment variable (scFv) antibody fragments and analyze their relative success, mainly in terms of their clinical performance. The use of scFv-based immunoconjugates, in combination with standard or novel therapies, is showing significant improvements in objective responses, suggesting that these reagents will become important components of the antineoplastic protocols for the future.

\section{HUMAN ANTIBODIES IN SCFV FORMAT DISPLAYED ON PHAGES AS CARRIER FOR THE CONSTRUCTION OF IMMUNOTHERAPEUTIC COMPOUNDS}

Clinical studies were initially focused on murine mAbs and on immunoconjugates that used radioactive elements or cytotoxins to kill tumor cells [29]. In this context two murine anti-CD20 mAbs have been conjugated with iodine-131 (131I) or yttritum-90 (90Y, beta-emitting radionuclides) to deliver tumoricidal doses of radiation to Non-Hodgkin's Lymphoma (NHL) patients whose disease had relapsed or had not responded to cytotoxic effect of Rituximab (anti CD20 chimeric antibody) [30]. These two radioimmunoconjugates, BEXXAR (131I) [31] and Zevalin (90Y) [32] have both been approved by international regulatory agency (FDA) and are currently used for eradication of NHL cells. However, murine mAbs may suffer from rapid formation of human anti-mouse antibodies (HAMA) that alter the pharmacokinetic profile of the antibody, leading to severe toxicity and preventing repeat dosing [33]. Historically, researchers have accepted the disadvantages of murine mAbs because of the impracticability of working with $\mathrm{mAbs}$ of human origin. At the end of ' 70 , the isolation of various human myeloma cell lines [34], and somatic cell genetics procedure for in vitro immortalization of human EBV infected B-cells raised a legitimate hope for the production of human hybridomas and/or stable B-cell line secreting mAbs of desired specificity [35]. The advantages of human mAbs, particularly their lack of immunogenicity, made them very attractive for diagnostic and therapeutic applications. However human hybridomas and EBV -immortalized B-cell lines are unfortunately characterized by poor and not stable $\mathrm{IgG}$ secretion. This biological aspect represents a relevant limitation in the harvesting homogeneous drug substance for medical preparations. The need for homogeneous sources of Abs with single antigen specificities therefore has promoted the search for alternative techniques to produce Abs useful in human therapy. Today, with the advances of recombinant genetic strategies rodent antibodies have been genetically modified in chimeric and humanized version significantly reducing their immunogenicity $[36,37]$. However, the main pitfalls of the use of conventional mAbs (usually $\operatorname{IgG}$ ) in cancer diagnosis and therapy, are related not only to their immunogenicity but also to the Abs large size $(150 \mathrm{kDa})$. This biochemical property is particularly relevant in solid tumors where physiological parameters such as imperfect vascular supply and increased interstitial pressure, inhibit the diffusion of larger molecules [38]. Moreover, the slow rates of clearance of intact mAbs due to their large size cause significant exposure to normal organs and limit the quantities delivered to tumors [39]. In this context, the ability to produce fully human antibody fragments has represented a significant breakthrough in the field of antibody engineering. Antibody fragments in particular in $\mathrm{scFv}$ format (, with their small size $(27 \mathrm{kDa})$, display better tumor penetration compared to full length monoclonal antibodies without any significant toxicity. After the introduction of the phage display technology originally described in 1985 [40] and 
thanks to the pioneering work of Winter and his co-workers [41], antibody fragments can now be isolated from libraries containing extensive repertoires displayed on the surface of filamentous bacteriophages by-passing hybridoma technology [42, 43]. From these scFv phage libraries, specific high-affinity antibodies with a particular phenotype (e.g. binding affinity) can be selected using a target antigen, thereby allowing simultaneous recovery of the gene encoding the selected antibody (Fig. 1). Frequently, the recovered gene can be subjected to cycles of mutation and further selection to enhance affinity. Furthermore, new methods of selection and screening have been designed to specifically isolate antibodies with desired characteristics, such as enhanced stability, high expression or capacity to activate the receptor [44]. ScFvs represent the smallest stable antibody fragments still capable of specifically binding an antigen. They are structured as a single polypeptide chain incorporating a heavy chain variable $(\mathrm{VH})$ and a light chain variable (VL) region of an antibody, linked by a flexible linker. The extremely versatile format of scFvs can be tailored by genetic engineering to improve affinity and stability. They can be modified in their size, pharmacokinetics, immunogenicity, specificity, valency and effectors functions [45]. Moreover, scFvs can be easily expressed and produced in E. coli in large quantity [46].
Human mAbs in scFv format have both pros and cons as therapeutics compared with full-size mAb. As discussed above, one advantage is that they are smaller and penetrate tissues and tumors more rapidly and deeply than intact mAbs. In addition, the small size of antibody fragments has been suggested to permit rescue of cryptic epitopes not accessible to full-sized mAbs [47]. However, scFvs are monovalent and often exhibit a rapid clearance with a half life in the range of few hours and poor retention time on the target [38]. Therefore, $\mathrm{scFv}_{\mathrm{v}}$ fragments have been engineered into dimeric, trimeric or tetrameric conjugates to slow down the clearance rate and to increase functional affinity, through the use of either chemical or genetic cross-links [48]. Of the various strategies to genetically encoding multimeric scFvs, the most successful design has been the reduction of $\mathrm{scFv}$ linker length between the VH and VL to $10-5$ residues in order to induce the formation of non-covalent dimers ("diabodies"; $55 \mathrm{kDa}$ ) [49], or trivalent trimers ("triabodies"; $90 \mathrm{kDa}$ ) or tetramers ("tetrabodies"; $120 \mathrm{kDa}$ ) if the linker length is reduced under 3 residues. Indeed, clinical trials of $\mathrm{scFv}$ fragments are likely to be as multivalent reagents or small immunoprotein fragments (SIP) constituted by two $\mathrm{scFv}$ linked together with an $\mathrm{Fc}$ fragment $(\mathrm{CH} 3$ or $\mathrm{CH} 4$ fragment) [50] (Fig. 2). For example, the fusion protein composed by SIP L19 and Interleukin 2 (L19-IL2)

\section{Isolation by panning of ScFv monoclonal antibody by phage antibody library}
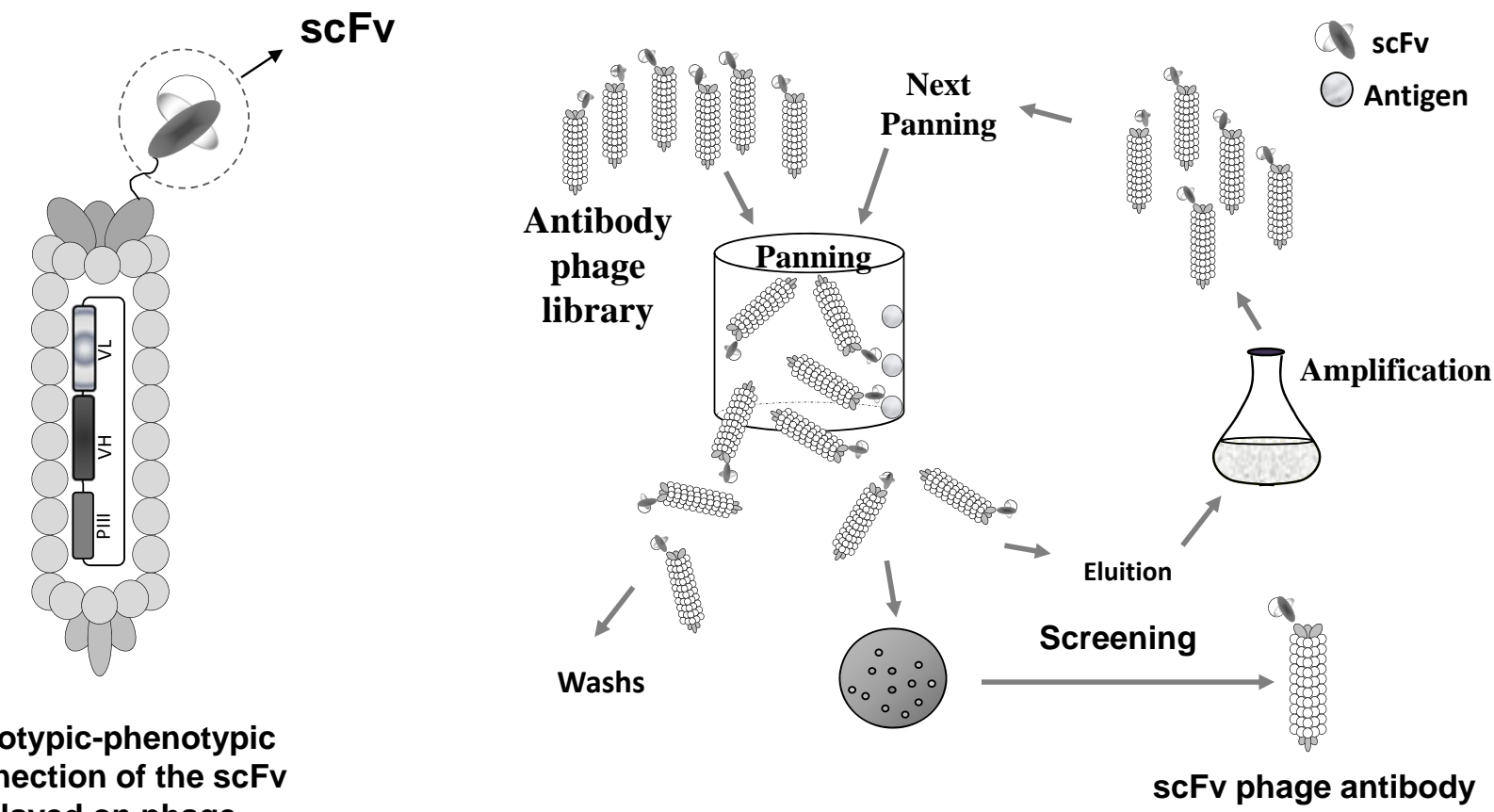
Genotypic-phenotypic connection of the ScFv displayed on phage

Fig. (1). Scheme of the isolation of monoclonal antibody in $\mathrm{scFv}$ format from antibody phage library. Library phage are first incubated with antigen immobilized on a solid surface. Specific phage bind and non-specific phage are washed away. The antigen-specific phage are eluted and used to re-infect bacteria for further rounds of selection and screening for antigen- specific phage. 


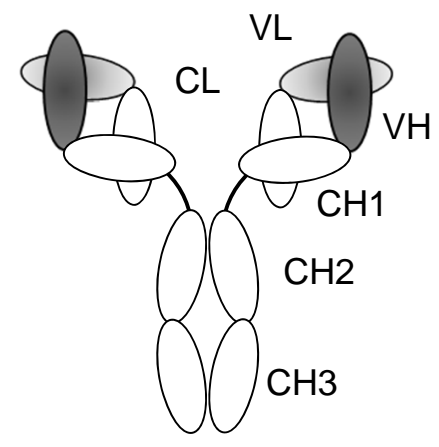

Classical IgG form of antibody

$(145-150 \mathrm{kDa})$

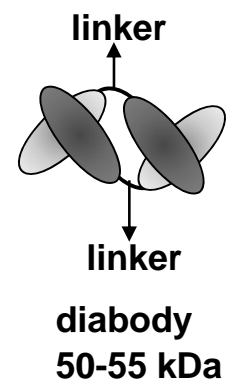

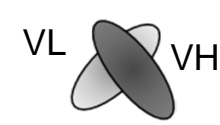

linker variable fragment

12-12.5 kDa single chain fragment variable (scFv)

27-30 kDa

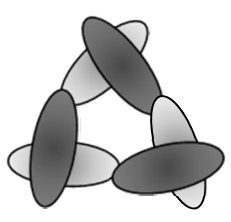

triabody 80-90 kDa

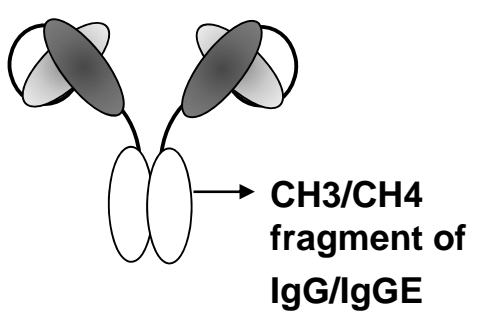

Small immunoprotein fragment
(SIP 75-80 kDa)

Fig. (2). Immunoglobulin and antibody fragments. Comparison of schematic biochemical structure between classical IgG immunoglobulin and antibody fragments generated by phage display and genetic recombination.

specifically directing to fibronectin and tenascin isoforms exhibits functional affinity and has been very successful in phaseI/II clinical studies of solid tumors in combination with conventional anticancer drugs [51-53].

Hence, there is an urgent need to rapidly develop therapeutic modalities that combine the advantages of specific tumor targeting of monoclonal antibodies fragments with curative molecules displaying potent and selective cytotoxic properties [54]. In this context, CEACAM1 is an attractive target for cancer immunotherapeutic strategies because of its expression profile, its role in the development of aggressive melanomas and carcinomas, and its expression in cancer metastasis. We have recently described the isolation and the affinity maturation by mutagenesis in vitro of a new anti-CEACAM1 $\mathrm{scFv}$ named E8, from an ETH-2 synthetic antibody library [55]. This human antibody recognizes a large panel of tumors including lung carcinomas and melanomas that over-express CEACAM1 protein on their cell surface. It is particularly interesting that scFvE8 shows no reactivity on various normal human cells and tissues [55].

The scFvE8 antibody which recognizes a common epitope distributed on CEACAM1, CEACAM3 and CEACAM5 meets all criteria for a biological compound to be used for diagnosis and therapy of cancer: it is human, hence poorly or not at all immunogenic, and it binds selectively to tumors expressing CEACAM1/5 cell adhesion molecules. Furthermore, to improve the pharmacokinetics and tumor retention of the scFvE8, oligomeric forms have been recently isolated by genetically modifying the structure of the antibody. This $\mathrm{scFv}$ variant named DIATHIS 1 maintains the specificity of the ancestral antibody and applying a novel purification procedure may be recovered as drug substance formed in a large percentage of stable oligomeric forms of $\mathrm{scFv}$ antibodies (paper in preparation).

In order to apply DIATHIS1 for immunotherapeutic strategies, we intend to pay particular attention to previous published methods reporting and discussing key methodologies to enhance antibody cytotoxicity by appending drugs, toxins, chemokines, enzymes or radionuclides to recombinant antibody fragments, generating antibody drug conjugates (ADCs) [56-58]. The tumor delivered cytotoxicity of many of these immunoconjugates has been directly related to their ability to enter the cell. Oligomeric forms of the scFv DIATHIS1 antibody provide a useful set of tools to construct human $\mathrm{scFv}$ mAbs with an appropriated PK/PD (Pharmacokinetic/Pharmacodynamic) for clinical development of tumor delivered curative agents. To this regard, preliminary studies conducted using the methods described by Ford et al., [59] indicate that the scFv DIATHIS1 is internalized in melanoma and adenocarcinoma (M. Cianfriglia, unpublished) permitting the design of a large 
array of immunoconjugates delivering their cytotoxic activity in tumor cells sparing normal tissues.

\section{SCFVS FOR DELIVERING CURATIVE PROTEINS TO NATURALLY CHEMORESISTANT TUMORS}

The outcome for patients with disseminated melanoma is poor and traditional chemotherapy is an extremely ineffective means of treating the natural drug-resistant characteristic of this disease [60-62]. However, a growing understanding of the molecular events that mediate tumor growth and metastatic spread has led to the development of rationally designed targeted therapeutics such as the antibody-mediated delivery of curative proteins to tumors. For the eradication of melanoma cells, Liu et al., [63], have experimented a fusion protein composed by single-chain antibody scFvMEL and the human granzyme B (GrB), a serine protease inducing apoptosis on target cells and working in combination with perforin (PFN). This scFvMEL, targets melanoma gp240 antigen and bound with melanoma cells while GrB exerts impressive cytotoxic effects by inducing apoptosis. The authors also evaluated the effects of GrB/scFvMEL on chemotherapy, radiation therapy, metastasis in vitro, and the growth of human melanoma A375 tumor xenografts in nude mice. GrB/scFvMEL showed synergistic cytotoxicity when coadministered with doxorubicin, vincristine or cisplatin, and additive effects, in combination with etoposide or cytarabine. Optimal cytotoxic effects were reported when cells were treated first with GrB/scFvMEL followed by exposure to the agent (rather than the reverse). Pretreatment of A375 cells with GrB/scFvMEL significantly sensitized melanoma cells to ionizing radiation. The results obtained by Liu and co workers [63] have proved the efficacy of the use of specific scFvs as carriers of effector proteins for the treatment of malignant melanoma. In this context, thanks to the specificity of $\mathrm{scFv}$ DIATHIS1 for metastatic melanoma cells, it would be interesting to design a similar fusion protein substituting scFvMEL with scFv DIATHIS1 and to verify the effector function of delivered GrB on melanoma cells following the multimodal therapeutic protocol used for A375 cells.

Markers of angiogenesis (e.g. CEACAM1) and of the tumor stroma represent particularly attractive targets for the antibody-based delivery of therapeutic agents. Recently, Marlind et al., [64], described that the immunocytokine F16IL2, consisting of the human monoclonal antibody F16 specific to the extradomain A1 of tenascin-C which is able to selectively accumulate at neovascular tumor sites, fused to human IL-2, displays a potent anticancer therapeutic activity. This activity is potentiated when the immunocytokine is administered in combination with chemotherapy in mice bearing human MDA-MB-231 breast tumors. The curative effect of specifically delivered IL-2 prompted us to genetically design and construct a fusion protein composed by the scFv DIATHIS1 and IL-2 to test its curative effect against CEACAM1 tumors in combination with taxanes and antracyclines (Mallano et al., in preparation).

\section{ANTIBODY DEPENDENT PRODRUG THERAPY (ADEPT)}

The ability of particular mAbs to specifically localize in tumor tissues in vivo offers a variety of therapeutic approaches for cancer therapy including delivery of specific enzymes which are then exploited to convert relatively non-toxic prodrugs into more active chemotherapeutic agents [65]. This is a two-step approach in which the antibody-enzyme complex is allowed to localize and clear from the circulation prior to the systemic administration of the prodrug. Thus, the prodrug is converted to an active chemotherapeutic agent by the enzyme which is principally located in the tumor tissue. Such an approach may overcome several limitations of the immuno-chemotherapy of tumors since the released drug can migrate throughout the tumor mass and exert activity on both the cells that have bound the enzyme conjugate and those that have not. Thus, one should be able to achieve a much higher concentration of drug within tumor masses while minimizing the toxic effects of the drug on non-target tissues [65]. Several excellent reviews have reported the potential of this two-step approach to cancer therapy $[66,67]$. The antimetabolite 5-fluorouracil (5-FU) is one of the most active chemotherapeutic agents for the treatment of colorectal cancer, but it has limited efficacy due to gastrointestinal and hematological toxicities [68]. High systemic toxicity of 5-FU could be circumvented by introducing gene directed enzyme prodrug therapy (GDEPT) and/or ADEPT relying on the ability of bacterial and/or yeast cytosine deaminase (yCD) enzyme to convert far less toxic substrate 5fluorocytosine (5-FC) to 5-FU [69]. Based on these premises, we have recently described the genetic construction and characterization of the ADEPT system consisting of the antiCEACAM1 scFvE8 fused to the yCD [70]. The scFvE8:yCD system showed the ability to render tumor cells susceptible to the far less toxic substrate 5-FC by its enzymatic conversion into 5-FU (Fig. 3). In vitro pre-treatment of malignant melanoma and gastric carcinoma (LoVo) cell lines with scFvE8:yCD followed by cell washing and incubation with 5FC, resulted in significant cell killing supporting the utility of this fusion protein as an agent for tumor-selective prodrug activation. The immunogenicity of the ADEPT system described in this study and that may severely interfere with the efficacy of this fusion protein, was not yet fully characterized. However, we are preparing a modified version of the fusion protein characterized by covalently attached PEG 5000 molecules [71]. Preliminary results show that this manipulation does not interfere with enzyme activity and abrogate the immunogenicity of the complex E8:yCD. Hence the ADEPT system herein described could become a new curative approach for cancer therapy. In this context, to allow the detection of $y C D$ expression at the protein level, we have also raised a human monoclonal antibody in $\mathrm{scFv}$ format against a recombinant $\mathrm{yCD}$ proved to be functionally active in NMR and in in vitro studies to convert the antifungal drug 5-FC into the anticancer compound 5-FU. The specificity of the human scFv was confirmed by Western blot and ELISA analyses. With this antibody, yCD expression can now be monitored without interfering with its enzymatic function in ADEPT and other studies leading to the effect of the so called tumor amplified protein expression and targeting to localize in vitro and in vivo generation of the anticancer agent 5-FU [72]

\section{ADCS IN THE THERAPY OF CEACAM1 POSITIVE TUMORS}

ADCs represent one of the most promising strategies to enhance the antitumor activity of antibodies. The therapeutic concept of ADCs is to use a mAb as a vehicle to deliver a 

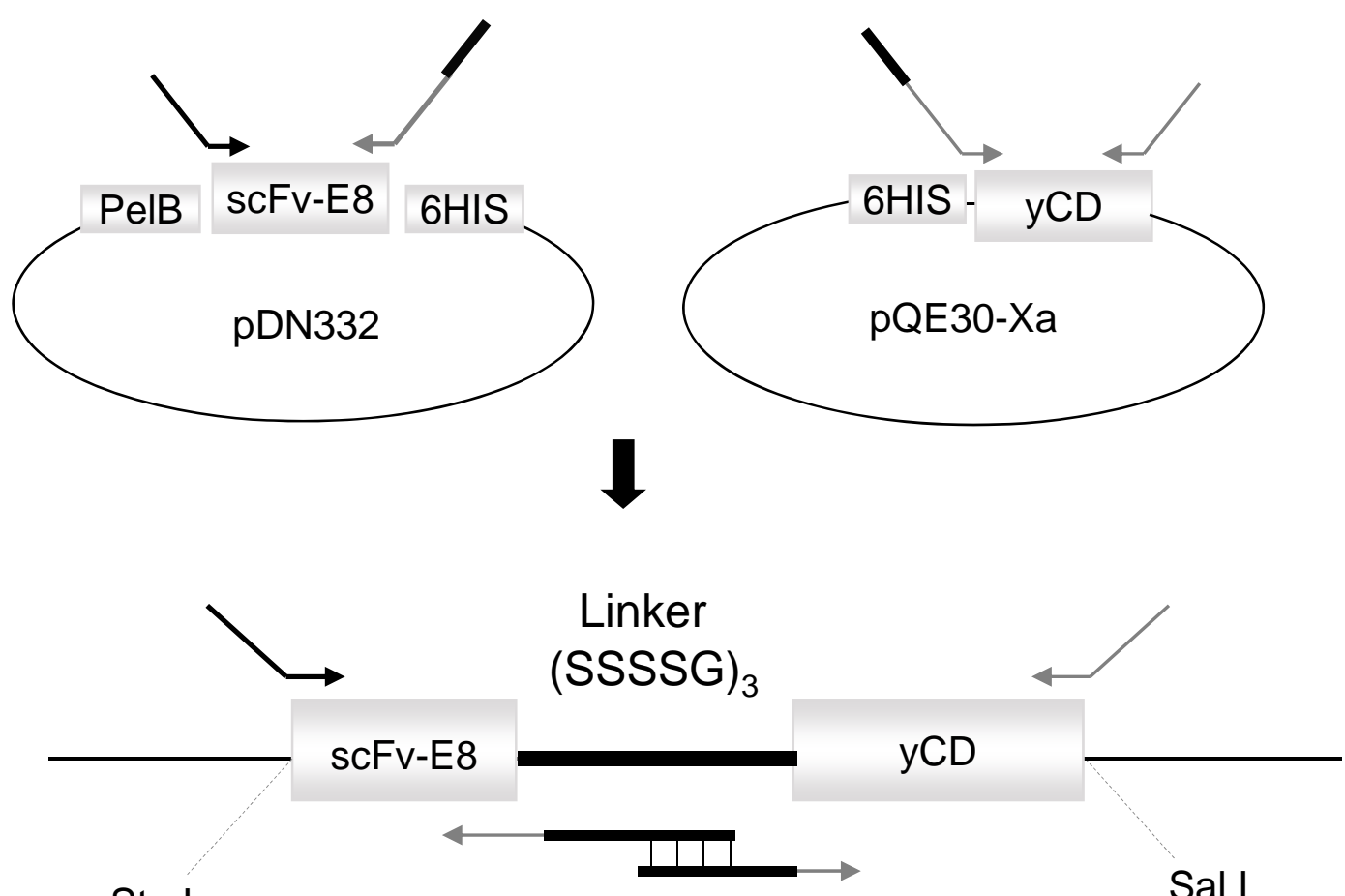

Stu I

Sal I

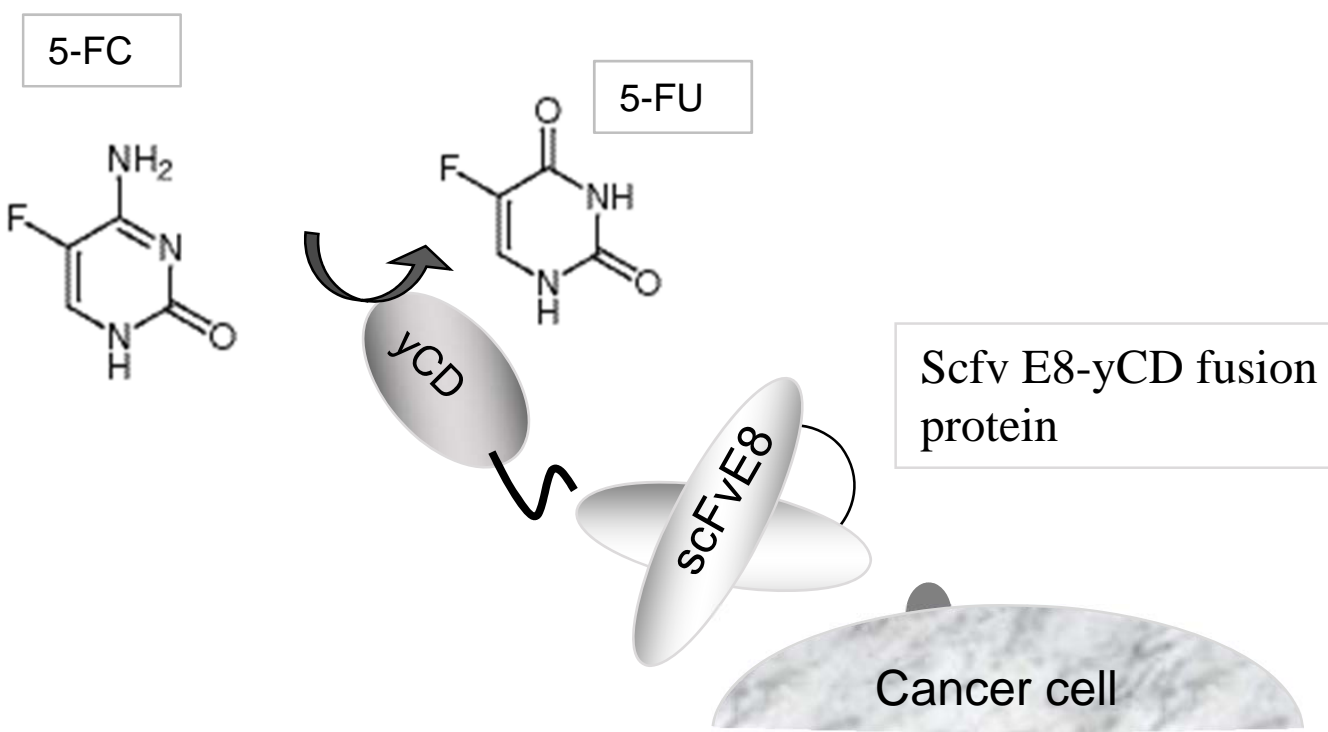

Fig. (3). Antibody depended prodrug enzyme therapy ( ADEPT ). Genetic design of the fusion protein constitute by the scFvE8 and Cytosine Deaminase. This construct is capable to convert in in vitro and in vivo system the inert antibiotic 5-Fluorocytosine (5-FC) in the potent cytotoxic compound 5-Fluorouracil (5-FU).

cytotoxic drug specifically to a tumor cell. As a consequence, ADCs have significant potential for enhancing the antitumor activity of 'naked' antibodies while reducing the systemic toxicity of the conjugated drug [73]. ADCs comprise an antibody (or an antibody fragment) conjugated to a cytotoxic drug via a chemical linker. Although such agents are simple in principle, deriving activities from them has been a major challenge because the mAb-conjugated drugs can decompose before being delivered to the target site, the pharmacokinetics and biodistribution of the $\mathrm{mAb}$ carriers may be suboptimal, the conjugation process can perturb $\mathrm{mAb}$ binding characteristics, the linkers used may have inappropriate stability and the drugs may not be released in active states.

In general, intact mAbs may not be the most favorable format for in vivo delivery of cytotoxic drugs and radionuclides to target tissues because their long circulating half-lives (typically days to weeks in the humanized version) result in high level of toxicity $[74,75]$. In contrast human antibodies in scFv format show much more rapid blood 
clearance properties therefore are more suitable for ADC applications. In particular, multivalent scFvs have been explored and have appeared to result in increased functional affinities and total tumor uptakes, compared to monovalent $\mathrm{scFv}$ molecule [76]. Multivalent $\mathrm{scFv}$ constructs in the molecular weight range of 60-120 kDa have shown superior avidity and tumor-targeting properties and present the ideal compromise between rapid pharmacokinetics and sufficient tumor accumulation required in applications such as radioimmunotheraphy (RIT) and ADC's. Early works in antibody-mediated drug targeting surrounded the use of clinically approved drugs, such as doxorubicin, methotrexate, mitomycin, 5-fluorouracil, and vinca alkaloid, since much was known about how they could be chemically manipulated and the agents were readily available. Little attention was paid to particular aspects of the $\mathrm{mAb}$ carrier, the mode and stoichiometry of drug attachment and the mechanism of drug release [54]. As a result, the activities of these agents were marginal, and few of them were extensively investigated [77]. Nonetheless, successful results were obtained by linking conventional drugs with specific human antibody in scFv format. Chen et al., [78] constructed the humanized anti-CEA scFv T84.66 and coupled it by dextran T-40 with mitomycin (MMC), a chemotherapeutic agent against colorectal cancer, and investigated the influence of this conjugate on the growth and apoptosis of colorectal cancer cells. The immunoreactivity of the conjugate against colorectal cancer cells was measured and confirmed by routinary laboratory techniques and functional assays demonstrating that this ADC inhibits growth and induces apoptosis on colorectal cancer in a dose dependent manner. However, the general low potency of ADCs carrying drugs such as methotrexate, mitomycin, 5fluorouracil, and vinca alkaloids prompted significant efforts towards utilizing drugs with much higher potencies able to kill tumor cells at concentrations well below that of standard chemotherapeutic agents.

To this regard, Kim et al., [79] genetically engineered an scFv-based anti CD30 diabody using cysteine residues for site-specific conjugation to monomethyl auristatin $\mathrm{E}$ (MMAE) or F (MMAF) demonstrating that this class of antibody fragments may be an effective immunoconjugated for delivering potent cytotoxic dugs to the tumor site.

\section{INNOVATIVE SCFV-BASED STRATEGIES FOR CANCER TREATMENT}

While there are several unresolved issues surrounding ADC's technology, such as the importance of antibody affinity, internalization rate, position and stoichiometry of drug substitution, and the acceptability of normal tissue antigen expression, it is apparent from the progresses in the past few years that ADCs will have an increasing impact in modeling novel and effective cancer treatments. Recently, the innovative curative potential of ADCs has been critically discussed at the World Antibody Drug Conjugate Summit Europe, organized by Biorbis/Hanson Wade in Frankfurt, Germany on February 21-23, 2011 [80]. In this meeting three new ADCs that are in late-stage clinical development, trastuzumab emtansine (T-DM1), brentuximab vedotin (SGN-35) and inotuzumab ozogamicin (CMC-544), were presented respectively by ImmunoGen, Genentech, Roche, Seattle Genetics and Pfizer. These ADCs have shown encouraging therapeutic effects against solid tumors (TDM1) and hematological malignancies (SGN-35, CMC544). In addition, data from new therapeutic ADC's entities and new process development strategies were discussed. For example, Algeta's lead product, which is based on radium223 , is a first-in-class, highly targeted alpha pharmaceutical under clinical evaluation for improvement of survival in patients with bone metastasis from advanced cancer [81]. Roland Kontermann from Stuttgart University illustrated how mAbs conjugated nanoparticles for targeted drug delivery with improved selectivity and therapeutic efficacy might be constructed using immuno-liposomes [82]. In this latter condition immuno-liposomes prepared from whole antibodies have been shown to be immunogenic and rapidly cleared from circulation through $\mathrm{Fc}$-mediated uptake by macrophages (Kupffer cells of the liver). These drawbacks can be circumvented using scFv molecules as ligands which lack the Fc fragment. ScFvs are small and can be easily modified through genetic engineering. Two approaches to generate immunoliposomes have been described in the literature: (1) the conventional method, in which antibody molecules are coupled directly to the liposome surface; and (2) the post-insertion method, where the ligands are first coupled to micelles prepared from functionalized lipids that are then inserted into preformed liposomes. The postinsertion method offers the advantage of independent liposome preparation (including drug loading) and $\mathrm{scFv}$ coupling, with each step performed under optimal conditions or even using commercially available drug-loaded liposomes such as Doxil (liposomal doxorubicin). In summary, liposomal drugs passively accumulate in tumors; insertion of ligands, e.g., genetically engineered antibody fragments such as the human scFv DIATHIS1, enables targeted delivery to tumor cells in vitro and in vivo. Efficient drug release (extra or intracellularly) is central to further improve the antibodyconjugated nanoparticles for targeted drug delivery.

\section{THE LESSON LEARNED FROM THE FIRST GENERATION OF ADCS AND THE IMPORTANCE OF PERSONALIZING CANCER CARE}

Gemtuzumab ozogamicin (Mylotarg; Pfizer), an antiCD33 humanized mAb conjugated to calicheamicin, is a heterogeneous mixture of $50 \%$ conjugated $(0$ to 8 calicheamicin moieties per $\operatorname{IgG}$ molecules, with an average of two or three, randomly linked to solvent-exposed lysyl residues of the antibody) and 50\% unconjugated antibody. It was approved by the US Food and Drug Administration (FDA) in 2000 for the treatment of patients with acute myeloid leukemia (AML). However, in June 2010, after post marketing reports and a new phase III clinical study, the agency and Pfizer, withdrew the drug from the market for the adverse events observed which include severe hepatic veno-occlusive disease (VOD) and death from liver failure in patients without a history of liver disease or hematopoietic stem cell transplant (HSCT) [83]. The generation of a biosimilar version of Mylotarg designed with a human $\mathrm{scFv}$ anti CD33 antibody for specifically delivering to AML cells cytotoxic compounds may offer a potential way to circumvent some of the dramatic pharmacokinetics limitations of the IgG4-calicheamicin drug conjugates. $\mathrm{ScFv}$ or derivative diabodies unlike $\mathrm{IgG}$, are eliminated rapidly and primarily by renal filtration. If antibody fragment drug 
conjugates are excreted with drug still attached, this will lessen the exposure of non tumor sites to free drug and potentially reduce the toxicity observed with IgG-drug conjugates [84]. Hence this novel form of $\mathrm{scFv}^{-b a s e d}$ ADCs model may show excellent safety pharmacokinetics profiles and therapeutic efficiency due to tumor penetration and sufficient retention time for releasing curative molecules.

However, efficacy and safety profiles to personalize an immuno-conjugate include a variety of biochemical and biological aspects such as the stability of the linker used to attach the drug to the antibody both in the systemic circulation and inside the target cell, and the effects that chemical modification have on pharmacokinetics, biodistribution, and antigen binding. Other important considerations are the choice of target antigen, the ability of the ADC to localized to target tissues, the fate of the antibody once bound to its cognate antigen, and the potency and mechanism of action of the released drug [54]. Even though all these methodological aspects were considered during the Mylotarg drug product development a series of concerns about the safety and efficacy emerging in clinical studies were under-evaluated since the number and complexity of the biological elements involved in gentuzumab ozogamicin ADC design. For example the multidrug resistance protein 1 (MDR1)-P glycoprotein, a multidrug transporter transmembrane protein that it is able to efflux out from cells a large variety of anticancer compound including calicheamicin, is expressed in a high percentage $(60 / 70 \%)$ of AML CD33 cells [85]. In contrast to the recommendation of experts [86] on the key role of MDR1Pglycoprotein expression in Mylotarg function, this multidrug transporter has been evaluated with a plethora of different mAbs some of them not truly specific [ 87, 88]. Further, MDR-P-glycoprotein efflux activity was evaluated with structurally different dyes substrate and MDR reversal agents. The cytotoxicity observed in vivo in CD33 negative cells during gentuzumab ozogamicin treatment suggest that the acid-labile hydrazone linker broke down in the blood stream allowing the drug to strike normal tissues and cells. Therefore, it is possible that non-specific drug release through linker instability contributes to the activities of gemtuzumab ozogamicin in non CD33 tumor cell types. It is possible that $\mathrm{mAb}$ drug conjugates using hydrazone linkers sensitive to acidic environment of the tumor cell readily deliver and liberate free drug or toxin within the target cell and yet are relatively unstable in circulation compared with the circulating half-life of the $\mathrm{mAb}$, resulting in premature drug release [80].

Further, the inter-patient differences in calicheamicin sensitivity are the largest differences in drug sensitivity ever observed in pediatric AML (more than 100 000-fold difference in calicheamicin sensitivity between the most sensitive and the most resistant patient samples) [89]. This dramatic difference in AML response to calicheamicin and the use of non-validated methodologies to evaluate level and function of MDR1-P-glycoprotein make tremendously difficult to identify an effective and safe therapeutic dosage of Gentuzumab ozogamicin in each single treated patient as well as the most effective concentration of MDR reversing agents (PSC833, Zosuquidar) to re-sensitize patients to the drug.
Despite this set-back, the extensive recorded data and the lessons learned for this first-in-class ADC helped to pave the way for the next generation immunoconjugates. At least 15 promising new ADCs are currently investigated in clinical trials and one of them, Brentuximab vedotin, has been recently approved for commercial distribution by the European Medicines Agency (EMA). Brentuximab vedotin is a chimeric anti-CD30 monoclonal antibody cAC10, linked to the antimitotic agents MMAE or MMAF, with potent and highly CD30-selective anti-tumor activity in vitro and in vivo [90]. This second generation ADCs employ linkers of protease-cleavable dipeptides. The drugs are appended via a valine-citrulline (vc) dipeptide linkage designed for high stability in serum and conditional cleavage and putative release of fully active drugs by lysosomal cathepsins. This drug linker system was shown to be highly stable in vitro and in vivo and when applied to multiple mAbs, the resulting ADCs were selectively potent and effective against cognate antigen-positive tumor cells and tumor xenografts $[79,91]$. This linker system has been also efficiently apply to anti $\mathrm{CD} 30$ phage antibodies in $\mathrm{scFv}$ format ( $\mathrm{scFv}$ diabodies were conjugated with 4 equivalents MMAE or MMAF, via a protease-cleavable dipeptide linker, to create the conjugates diabody-vcE4 and diabody-vcF4, respectively) [79]. This third generation of ADCs may offer a potential way to circumvent some of pharmacokinetics limitations of IgG drug conjugates.

All scFv based strategies so far designed may have a great curative potential. Nonetheless their safety and efficacy should be demonstrated in appropriated preclinical models and in presence of severe interpretation in clinical studies. The withdrawn of Mylotrag from the market has a huge social, ethical and psychological implications for patients and their families and represents relevant concerns versus the positivistic curative attitude of cancer-basedimmunotherapy. Nonetheless, these failures should be also considered as an important opportunity to design innovative and personalized antibody -based immunotherapies with more appropriated safety and efficacy profiles.

\section{CONCLUSION AND PERSPECTIVES}

The evidence that the human antibody DIATHIS1 in $\mathrm{scFv}$ format recognizes with exquisite specificity a cell surface epitope in the CEACAM1 antigen in malignant melanoma and Non-small-cell lung carcinoma (NSCLC) prompted us to use such antibody as vehicle for radionuclides (typically ß-emitters) and biological molecules with curative potential such as IL2. The principal aim of radionuclide studies is to achieve better imaging and therapy of cancer. In the ideal case, a cancer patient would first receive a diagnostic dose of the antibody labeled with a radionuclide compatible with imaging procedures (e.g., single photon emission computed tomography (SPECT) or positron emission tomography (PET). If adequate antibody localization at the site of disease is achieved, the patient could receive a therapeutic dose of the same antibody labeled with a radionuclide capable of inducing curative effects. Although RIT has been proven successful in the treatment of haematologic malignancies, the treatment of solid tumors, has proven more difficult, with a number of factors, such as the reduced radiosensitivity, limiting therapeutic efficacy. Particular limitations of RIT include bone marrow toxicity 
resulting from the long-circulating half-life of radiolabeled mAbs, as well as the heterogeneous penetration (binding site barrier) and irradiation of tumors [58]. Similar issues have arisen when drugs and toxins have been conjugated to IgG mAbs in cancer therapy. In an effort to overcome these limitations we have propose in this review the utilization of specific scFvs produced by joining the genes for the $\mathrm{VH}$ and VL domains of an antibody with a short oligonucleotide linker between them to prevent dissociation [92]. The reduced size and lack of $\mathrm{Fc}$ domain of $\mathrm{scFv}$ antibody constructs result in faster pharmacokinetics and potentially more homogenous tumor penetration relative to large $\mathrm{IgG}$ molecules.

The usefulness of phage antibody libraries as a tool for generating scFvs against tumor associated antigens has been largely used in the past 5 years and more than 50 human antibodies developed with this technique are under clinical evaluation for safety and efficacy in the treatment of several pathologies including cancers. The efficient display of recombinant antibodies on filamentous phage has been also assessed by our findings showing that soluble human scFvs selected from the ETH-2 library may yield agents capable of specifically recognizing metastatic melanoma, breast, colon and lung cancer [55]. The human DIATHIS1 antibody recognizing with exquisite specificity an epitope shared by CEACAM1/3/5 meets all criteria for a biological compound to be used for diagnosis and therapy of cancer: it is human, hence poorly or not at all immunogenic, and it binds selectively to CEACAM1/5 cell surface antigens expressed on melanoma, NSCLC and other solid tumors naturally refractory to chemoterapeutic treatment. Furthermore, its small molecular size should provide for efficient tissue penetration, yet give high efficacy in solid tumor treatment and excellent safety profiles for rapid plasma clearance.

\section{ACKNOWLEDGEMENTS}

The present research article has been supported with intramural funds of Italian National Institute of Health (ISS).

\section{CONFLICT OF INTEREST}

Declared none.

\section{REFERENCES}

[1] Beauchemin, N.; Draber, P.; Dveksler, G.; Gold, P.; Gray-Owen, S.; Grunert, F.; Hammarstrom, S.; Holmes, K. V.; Karlsson, A.; Kuroki, M.; Lin, S. H.; Lucka, L.; Najjar, S. M.; Neumaier, M.; Obrink, B.; Shively, J. E.; Skubitz, K. M.; Stanners, C. P.; Thomas, P.; Thompson, J. A.; Virji, M.; von, K. S.; Wagener, C.; Watt, S.; Zimmermann, W. Redefined nomenclature for members of the carcinoembryonic antigen family. Exp. Cell Res., 1999, 252 (2), 243-249.

[2] Deangelis, A. M.; Heinrich, G.; Dai, T.; Bowman, T. A.; Patel, P. R.; Lee, S. J.; Hong, E. G.; Jung, D. Y.; Assmann, A.; Kulkarni, R. N.; Kim, J. K.; Najjar, S. M. Carcinoembryonic antigen-related cell adhesion molecule 1: a link between insulin and lipid metabolism. Diabetes, 2008, 57 (9), 2296-2303.

[3] Wagener, C.; Ergun, S. Angiogenic properties of the carcinoembryonic antigen-related cell adhesion molecule 1. Exp. Cell Res., 2000, 261 (1), 19-24.

[4] Gray-Owen, S. D.; Blumberg, R. S. CEACAM1: contact-dependent control of immunity. Nat. Rev. Immunol., 2006, 6 (6), 433-446.

[5] Voges, M.; Bachmann, V.; Kammerer, R.; Gophna, U.; Hauck, C. R. CEACAM1 recognition by bacterial pathogens is speciesspecific. BMC. Microbiol., 2010, 10, 117.

[6] Gold, P.; Freedman, S. O. Specific carcinoembryonic antigens of the human digestive system. J. Exp. Med., 1965, 122 (3), 467-481.
[7] Hammarstrom, S. The carcinoembryonic antigen (CEA) family: structures, suggested functions and expression in normal and malignant tissues. Semin. Cancer Biol., 1999, 9 (2), 67-81.

[8] Kuespert, K.; Pils, S.; Hauck, C. R. CEACAMs: their role in physiology and pathophysiology. Curr. Opin. Cell Biol., 2006, 18 (5), 565-571.

[9] Watt, S. M.; Teixeira, A. M.; Zhou, G. Q.; Doyonnas, R.; Zhang, Y.; Grunert, F.; Blumberg, R. S.; Kuroki, M.; Skubitz, K. M.; Bates, P. A. Homophilic adhesion of human CEACAM1 involves N-terminal domain interactions: structural analysis of the binding site., Blood 2001, 98 (5), 1469-1479.

[10] Stern, N.; Markel, G.; Arnon, T. I.; Gruda, R.; Wong, H.; GrayOwen, S. D.; Mandelboim, O. Carcinoembryonic antigen (CEA) inhibits NK killing via interaction with CEA-related cell adhesion molecule 1. J. Immunol., 2005, 174 (11), 6692-6701.

[11] Thies, A.; Moll, I.; Berger, J.; Wagener, C.; Brummer, J.; Schulze, H. J.; Brunner, G.; Schumacher, U. CEACAM1 expression in cutaneous malignant melanoma predicts the development of metastatic disease. J. Clin. Oncol., 2002, 20 (10), 2530-2536.

[12] Laack, E.; Nikbakht, H.; Peters, A.; Kugler, C.; Jasiewicz, Y.; Edler, L.; Brummer, J.; Schumacher, U.; Hossfeld, D. K. Expression of CEACAM1 in adenocarcinoma of the lung: a factor of independent prognostic significance. J. Clin. Oncol., 2002, 20 (21), 4279-4284.

[13] Thom, I.; Schult-Kronefeld, O.; Burkholder, I.; Schuch, G.; Andritzky, B.; Kastendieck, H.; Edler, L.; Wagener, C.; Bokemeyer, C.; Schumacher, U.; Laack, E. Expression of CEACAM-1 in pulmonary adenocarcinomas and their metastases. Anticancer Res., 2009, 29 (1), 249-254.

[14] Tilki, D.; Irmak, S.; Oliveira-Ferrer, L.; Hauschild, J.; Miethe, K.; Atakaya, H.; Hammerer, P.; Friedrich, M. G.; Schuch, G.; Galalae, R.; Stief, C. G.; Kilic, E.; Huland, H.; Ergun, S. CEA-related cell adhesion molecule-1 is involved in angiogenic switch in prostate cancer. Oncogene, 2006, 25 (36), 4965-4974.

[15] Zhou, C. J.; Liu, B.; Zhu, K. X.; Zhang, Q. H.; Zhang, T. G.; Xu, W. H.; Wang, H. B.; Yu, W. H.; Qu, Y. D.; Wang, H. J.; Wu, H. L.; Sun, S. Z.; Guo, J. Q. The different expression of carcinoembryonic antigen-related cell adhesion molecule 1 (CEACAM1) and possible roles in gastric carcinomas. Pathol. Res. Pract., 2009, 205 (7), 483-489.

[16] Ieda, J.; Yokoyama, S.; Tamura, K.; Takifuji, K.; Hotta, T.; Matsuda, K.; Oku, Y.; Nasu, T.; Kiriyama, S.; Yamamoto, N.; Nakamura, Y.; Shively, J. E.; Yamaue, H. Re-expression of CEACAM1 long cytoplasmic domain isoform is associated with invasion and migration of colorectal cancer. Int. J. Cancer, 2011, 129 (6), 1351-1361.

[17] Kang, W. Y.; Chen, W. T.; Wu, M. T.; Chai, C. Y. The expression of CD66a and possible roles in colorectal adenoma and adenocarcinoma. Int. J. Colorectal Dis., 2007, 22 (8), 869-874.

[18] Ergun, S.; Kilik, N.; Ziegeler, G.; Hansen, A.; Nollau, P.; Gotze, J.; Wurmbach, J. H.; Horst, A.; Weil, J.; Fernando, M.; Wagener, C. CEA-related cell adhesion molecule 1: a potent angiogenic factor and a major effector of vascular endothelial growth factor. Mol. Cell, 2000, 5 (2), 311-320.

[19] Dango, S.; Sienel, W.; Schreiber, M.; Stremmel, C.; Kirschbaum, A.; Pantel, K.; Passlick, B. Elevated expression of carcinoembryonic antigen-related cell adhesion molecule 1 (CEACAM-1) is associated with increased angiogenic potential in non-small-cell lung cancer. Lung Cancer, 2008, 60 (3), 426-433.

[20] Markel, G.; Lieberman, N.; Katz, G.; Arnon, T. I.; Lotem, M.; Drize, O.; Blumberg, R. S.; Bar-Haim, E.; Mader, R.; Eisenbach, L.; Mandelboim, O. CD66a interactions between human melanoma and NK cells: a novel class I MHC-independent inhibitory mechanism of cytotoxicity. J. Immunol., 2002, 168 (6), 2803-2810.

[21] Markel, G.; Wolf, D.; Hanna, J.; Gazit, R.; Goldman-Wohl, D.; Lavy, Y.; Yagel, S.; Mandelboim, O. Pivotal role of CEACAM1 protein in the inhibition of activated decidual lymphocyte functions. J. Clin. Invest., 2002, 110 (7), 943-953.

[22] Markel, G.; Seidman, R.; Stern, N.; Cohen-Sinai, T.; Izhaki, O.; Katz, G.; Besser, M.; Treves, A. J.; Blumberg, R. S.; Loewenthal, R.; Mandelboim, O.; Orenstein, A.; Schachter, J. Inhibition of human tumor-infiltrating lymphocyte effector functions by the homophilic carcinoembryonic cell adhesion molecule 1 interactions. J. Immunol., 2006, 177 (9), 6062-6071.

[23] Markel, G.; Seidman, R.; Cohen, Y.; Besser, M. J.; Sinai, T. C.; Treves, A. J.; Orenstein, A.; Berger, R.; Schachter, J. Dynamic 
expression of protective CEACAM1 on melanoma cells during specific immune attack. Immunology, 2009, 126 (2), 186-200.

[24] Ryan, J. L.; Krishnan, S.; Movsas, B.; Coleman, C. N.; Vikram, B.; Yoo, S. S. Decreasing the adverse effects of cancer therapy: an NCI Workshop on the preclinical development of radiation injury mitigators/protectors. Radiat. Res., 2011, 176 (5), 688-691.

[25] Gottesman, M. M.; Fojo, T.; Bates, S. E. Multidrug resistance in cancer: role of ATP-dependent transporters. Nat. Rev. Cancer, 2002, 2 (1), 48-58.

[26] Sharkey, R. M.; Goldenberg, D. M. Targeted therapy of cancer: new prospects for antibodies and immunoconjugates. CA Cancer $J$. Clin., 2006, 56 (4), 226-243.

[27] Reichert, J. M.; Valge-Archer, V. E. Development trends for monoclonal antibody cancer therapeutics. Nat. Rev. Drug Discov., 2007, 6 (5), 349-356.

[28] Beck, A.; Haeuw, J. F.; Wurch, T.; Goetsch, L.; Bailly, C.; Corvaia, N. The next generation of antibody-drug conjugates comes of age. Discov. Med., 2010, 10 (53), 329-339.

[29] Milenic, D. E.; Brady, E. D.; Brechbiel, M. W. Antibody-targeted radiation cancer therapy. Nat. Rev. Drug Discov., 2004, 3 (6), 488499.

[30] Boye, J.; Elter, T.; Engert, A. An overview of the current clinical use of the anti-CD20 monoclonal antibody rituximab. Ann. Oncol., 2003, 14 (4), 520-535.

[31] Wahl, R. L. Tositumomab and (131)I therapy in non-Hodgkin's lymphoma. J. Nucl. Med., 2005, 46 (Suppl 1), 128S-140S.

[32] Borghaei, H.; Schilder, R. J. Safety and efficacy of radioimmunotherapy with yttrium 90 ibritumomab tiuxetan (Zevalin). Semin. Nucl. Med., 2004, 34 (1 Suppl 1), 4-9.

[33] Mirick, G. R.; Bradt, B. M.; Denardo, S. J.; Denardo, G. L. A review of human anti-globulin antibody (HAGA, HAMA, HACA, HAHA) responses to monoclonal antibodies. Not four letter words. Q. J. Nucl. Med. Mol. Imaging, 2004, 48 (4), 251-257.

[34] Olsson, L.; Kaplan, H. S. Human-human hybridomas producing monoclonal antibodies of predefined antigenic specificity. Proc. Natl. Acad. Sci. U. S. A., 1980, 77 (9), 5429-5431.

[35] Lanzavecchia, A.; Corti, D.; Sallusto, F. Human monoclonal antibodies by immortalization of memory B cells. Curr. Opin. Biotechnol., 2007, 18 (6), 523-528.

[36] Krebs, B.; Rauchenberger, R.; Reiffert, S.; Rothe, C.; Tesar, M.; Thomassen, E.; Cao, M.; Dreier, T.; Fischer, D.; Hoss, A.; Inge, L.; Knappik, A.; Marget, M.; Pack, P.; Meng, X. Q.; Schier, R.; Sohlemann, P.; Winter, J.; Wolle, J.; Kretzschmar, T. Highthroughput generation and engineering of recombinant human antibodies. J. Immunol. Methods, 2001, 254 (1-2), 67-84.

[37] Presta, L. G. Selection, design, and engineering of therapeutic antibodies. J. Allergy Clin. Immunol., 2005, 116 (4), 731-736.

[38] Beckman, R. A.; Weiner, L. M.; Davis, H. M. Antibody constructs in cancer therapy: protein engineering strategies to improve exposure in solid tumors. Cancer, 2007, 109 (2), 170-179.

[39] Breedveld, F. C. Therapeutic monoclonal antibodies. Lancet, 2000, 355 (9205), 735-740.

[40] Smith, G. P. Filamentous fusion phage: novel expression vectors that display cloned antigens on the virion surface. Science, 1985, 228 (4705), 1315-1317.

[41] Winter, G.; Griffiths, A. D.; Hawkins, R. E.; Hoogenboom, H. R. Making antibodies by phage display technology. Annu. Rev. Immunol., 1994, 12, 433-455.

[42] Nilsson, F.; Tarli, L.; Viti, F.; Neri, D. The use of phage display for the development of tumour targeting agents. Adv. Drug Deliv. Rev., 2000, 43 (2-3), 165-196.

[43] Hoogenboom, H. R. Selecting and screening recombinant antibody libraries. Nat. Biotechnol., 2005, 23 (9), 1105-1116.

[44] Hudson, P. J.; Souriau, C. Engineered antibodies. Nat. Med., 2003, $9(1), 129-134$.

[45] Accardi, L.; Di, B. P. Antibodies in single-chain format against tumour-associated antigens: present and future applications. Curr. Med. Chem., 2010, 17 (17), 1730-1755.

[46] Kipriyanov, S. M.; Little, M. Generation of recombinant antibodies. Mol. Biotechnol., 1999, 12 (2), 173-201.

[47] Henderikx, P.; Kandilogiannaki, M.; Petrarca, C.; von MensdorffPouilly, S.; Hilgers, J. H.; Krambovitis, E.; Arends, J. W.; Hoogenboom, H. R. Human single-chain Fv antibodies to MUC1 core peptide selected from phage display libraries recognize unique epitopes and predominantly bind adenocarcinoma. Cancer Res., 1998, 58 (19), 4324-4332.
[48] Dolezal, O.; De, G. R.; Walter, M.; Doughty, L.; Hattarki, M.; Hudson, P. J.; Kortt, A. A. Single-chain Fv multimers of the antineuraminidase antibody NC10: the residue at position 15 in the $\mathrm{V}(\mathrm{L})$ domain of the $\mathrm{scFv}^{-0}(\mathrm{~V}(\mathrm{~L})-\mathrm{V}(\mathrm{H}))$ molecule is primarily responsible for formation of a tetramer-trimer equilibrium. Protein. Eng., 2003, 16 (1), 47-56.

[49] Holliger, P.; Prospero, T.; Winter, G. "Diabodies": small bivalent and bispecific antibody fragments. Proc. Natl. Acad. Sci. U. S. A., 1993, 90 (14), 6444-6448.

[50] Borsi, L.; Balza, E.; Bestagno, M.; Castellani, P.; Carnemolla, B.; Biro, A.; Leprini, A.; Sepulveda, J.; Burrone, O.; Neri, D.; Zardi, L. Selective targeting of tumoral vasculature: comparison of different formats of an antibody (L19) to the ED-B domain of fibronectin. Int. J. Cancer, 2002, 102 (1), 75-85.

[51] Schliemann, C.; Palumbo, A.; Zuberbuhler, K.; Villa, A.; Kaspar, M.; Trachsel, E.; Klapper, W.; Menssen, H. D.; Neri, D. Complete eradication of human B-cell lymphoma xenografts using rituximab in combination with the immunocytokine L19-IL2. Blood, 2009, $113(10), 2275-2283$.

[52] Eigentler, T. K.; Weide, B.; de, B. F.; Spitaleri, G.; Romanini, A.; Pflugfelder, A.; Gonzalez-Iglesias, R.; Tasciotti, A.; Giovannoni, L.; Schwager, K.; Lovato, V.; Kaspar, M.; Trachsel, E.; Menssen, H. D.; Neri, D.; Garbe, C. A dose-escalation and signal-generating study of the immunocytokine L19-IL2 in combination with dacarbazine for the therapy of patients with metastatic melanoma. Clin. Cancer Res., 2011, 17 (24), 7732-7742.

[53] Johannsen, M.; Spitaleri, G.; Curigliano, G.; Roigas, J.; Weikert, S.; Kempkensteffen, C.; Roemer, A.; Kloeters, C.; Rogalla, P.; Pecher, G.; Miller, K.; Berndt, A.; Kosmehl, H.; Trachsel, E.; Kaspar, M.; Lovato, V.; Gonzalez-Iglesias, R.; Giovannoni, L.; Menssen, H. D.; Neri, D.; de, B. F. The tumour-targeting human L19-IL2 immunocytokine: preclinical safety studies, phase I clinical trial in patients with solid tumours and expansion into patients with advanced renal cell carcinoma. Eur. J. Cancer, 2010, 46 (16), 2926-2935.

[54] Senter, P. D. Potent antibody drug conjugates for cancer therapy. Curr. Opin. Chem. Biol., 2009, 13 (3), 235-244.

[55] Pavoni, E.; Flego, M.; Dupuis, M. L.; Barca, S.; Petronzelli, F.; Anastasi, A. M.; D'Alessio, V.; Pelliccia, A.; Vaccaro, P.; Monteriu, G.; Ascione, A.; De, S. R.; Felici, F.; Cianfriglia, M.; Minenkova, O. Selection, affinity maturation, and characterization of a human scFv antibody against CEA protein. BMC. Cancer, 2006, $6,41$.

[56] Kovtun, Y. V.; Goldmacher, V. S. Cell killing by antibody-drug conjugates. Cancer Lett., 2007, 255 (2), 232-240.

[57] Chari, R. V. Targeted cancer therapy: conferring specificity to cytotoxic drugs. Acc. Chem. Res., 2008, 41 (1), 98-107.

[58] Steiner, M.; Neri, D. Antibody-radionuclide conjugates for cancer therapy: historical considerations and new trends. Clin. Cancer Res., 2011, 17 (20), 6406-6416.

[59] Ford, C. H.; Tsaltas, G. C.; Osborne, P. A.; Addetia, K. Novel flow cytometric analysis of the progress and route of internalization of a monoclonal anti-carcinoembryonic antigen (CEA) antibody. Cytometry, 1996, 23 (3), 228-240.

[60] Mouawad, R.; Sebert, M.; Michels, J.; Bloch, J.; Spano, J. P.; Khayat, D. Treatment for metastatic malignant melanoma: old drugs and new strategies. Crit. Rev. Oncol. Hematol., 2010, 74 (1), 27-39.

[61] Mansfield, A. S.; Markovic, S. N. Novel therapeutics for the treatment of metastatic melanoma. Future Oncol., 2009, 5 (4), 543557.

[62] Sapoznik, S.; Ortenberg, R.; Schachter, J.; Markel, G. CEACAM1 in Malignant Melanoma: A Diagnostic and Therapeutic Target. Curr. Top. Med. Chem., 2012, 12(1), 3-10.

[63] Liu, Y.; Zhang, W.; Niu, T.; Cheung, L. H.; Munshi, A.; Meyn, R. E., Jr.; Rosenblum, M. G. Targeted apoptosis activation with $\mathrm{GrB} / \mathrm{scFvMEL}$ modulates melanoma growth, metastatic spread, chemosensitivity, and radiosensitivity. Neoplasia, 2006, 8 (2), 125135 .

[64] Marlind, J.; Kaspar, M.; Trachsel, E.; Sommavilla, R.; Hindle, S.; Bacci, C.; Giovannoni, L.; Neri, D. Antibody-mediated delivery of interleukin-2 to the stroma of breast cancer strongly enhances the potency of chemotherapy. Clin. Cancer Res., 2008, 14 (20), 65156524. 
[65] Bagshawe, K. D. Antibody-directed enzyme prodrug therapy (ADEPT) for cancer. Expert. Rev. Anticancer Ther., 2006, 6 (10), 1421-1431.

[66] Waldmann, T. A. Immunotherapy: past, present and future. Nat. Med., 2003, 9 (3), 269-277.

[67] Melton, R. G.; Sherwood, R. F. Antibody-enzyme conjugates for cancer therapy. J. Natl. Cancer Inst., 1996, 88 (3-4), 153-165.

[68] Douillard, J. Y.; Cunningham, D.; Roth, A. D.; Navarro, M.; James, R. D.; Karasek, P.; Jandik, P.; Iveson, T.; Carmichael, J.; Alakl, M.; Gruia, G.; Awad, L.; Rougier, P. Irinotecan combined with fluorouracil compared with fluorouracil alone as first-line treatment for metastatic colorectal cancer: a multicentre randomised trial. Lancet, 2000, 355 (9209), 1041-1047.

[69] Wallace, P. M.; MacMaster, J. F.; Smith, V. F.; Kerr, D. E.; Senter, P. D.; Cosand, W. L. Intratumoral generation of 5-fluorouracil mediated by an antibody-cytosine deaminase conjugate in combination with 5-fluorocytosine. Cancer Res., 1994, 54 (10), 2719-2723.

[70] Zamboni, S.; Mallano, A.; Flego, M.; Ascione, A.; Dupuis, M. L.; Gellini, M.; Barca, S.; Cianfriglia, M. Genetic construction, expression, and characterization of a single chain anti-CEA antibody fused to cytosine deaminase from yeast. Int. J. Oncol., 2008, 32 (6), 1245-1251.

[71] Abuchowski, A.; McCoy, J. R.; Palczuk, N. C.; van, E. T.; Davis, F. F. Effect of covalent attachment of polyethylene glycol on immunogenicity and circulating life of bovine liver catalase. $J$. Biol. Chem., 1977, 252 (11), 3582-3586.

[72] Mallano, A.; Zamboni, S.; Carpinelli, G.; Santoro, F.; Flego, M.; Ascione, A.; Gellini, M.; Tombesi, M.; Podo, F.; Cianfriglia, M. Generation and characterization of a human single-chain fragment variable (scFv) antibody against cytosine deaminase from Yeast. BMC. Biotechnol., 2008, 8, 68 .

[73] Carter, P. J.; Senter, P. D. Antibody-drug conjugates for cancer therapy. Cancer J., 2008, 14 (3), 154-169.

[74] Liu, X. Y.; Pop, L. M.; Vitetta, E. S. Engineering therapeutic monoclonal antibodies. Immunol. Rev., 2008, 222, 9-27.

[75] Lambert, J. M. Drug-conjugated monoclonal antibodies for the treatment of cancer. Curr. Opin. Pharmacol., 2005, 5 (5), 543-549.

[76] Kelly, M. P.; Lee, F. T.; Tahtis, K.; Power, B. E.; Smyth, F. E.; Brechbiel, M. W.; Hudson, P. J.; Scott, A. M. Tumor targeting by a multivalent single-chain $\mathrm{Fv}$ (scFv) anti-Lewis $\mathrm{Y}$ antibody construct. Cancer Biother. Radiopharm., 2008, 23 (4), 411-423.

[77] McDonagh, C. F.; Turcott, E.; Westendorf, L.; Webster, J. B.; Alley, S. C.; Kim, K.; Andreyka, J.; Stone, I.; Hamblett, K. J.; Francisco, J. A.; Carter, P. Engineered antibody-drug conjugates with defined sites and stoichiometries of drug attachment. Protein. Eng. Des. Sel., 2006, 19 (7), 299-307.

[78] Chen, D. J.; Tan, Z.; Chen, F.; Du, T. Construction of humanized carcinoembryonic antigen specific single chain variable fragment and mitomycin conjugate. World J. Gastroenterol., 2007, 13 (43), $5765-5770$.

[79] Kim, K. M.; McDonagh, C. F.; Westendorf, L.; Brown, L. L.; Sussman, D.; Feist, T.; Lyon, R.; Alley, S. C.; Okeley, N. M.; Zhang, X.; Thompson, M. C.; Stone, I.; Gerber, H. P.; Carter, P. J. Anti-CD30 diabody-drug conjugates with potent antitumor activity. Mol. Cancer Ther., 2008, 7 (8), 2486-2497.

[80] Beck, A.; Senter, P.; Chari, R. World Antibody Drug Conjugate Summit Europe: February 21-23, 2011; Frankfurt, Germany. MAbs. 2011, 3 (4), 331-337.
[81] Nilsson, S.; Larsen, R. H.; Fossa, S. D.; Balteskard, L.; Borch, K. W.; Westlin, J. E.; Salberg, G.; Bruland, O. S. First clinical experience with alpha-emitting radium-223 in the treatment of skeletal metastases. Clin. Cancer Res., 2005, 11 (12), 4451-4459.

[82] Rothdiener, M.; Beuttler, J.; Messerschmidt, S. K.; Kontermann, R. E. Antibody targeting of nanoparticles to tumor-specific receptors: immunoliposomes. Methods Mol. Biol., 2010, 624, 295-308.

[83] Ricart, AD. Antibody-drug conjugates of calicheamicin derivative: gemtuzumab ozogamicin and inotuzumab ozogamicin. Clin Cancer Res., 2011, 17(20), 6417-6427.

[84] Batra, S. K.; Jain, M.; Wittel, U. A.; Chauhan, S. C.; Colcher, D. Pharmacokinetics and biodistribution of genetically engineered antibodies. Curr. Opin. Biotechnol., 2002, 13 (6), 603-608.

[85] Cianfriglia, M.; Mallano, A.; Ascione, A.; Dupuis, M. L. Multidrug transporter proteins and cellular factors involved in free and $\mathrm{mAb}$ linked calicheamicin-gamma1 (gentuzumab ozogamicin, GO) resistance and in the selection of GO resistant variants of the HL60 AML cell line. Int. J. Oncol., 2010, 36 (6), 1513-1520.

[86] Leith, C. P.; Kopecky, K. J.; Chen, I. M.; Eijdems, L.; Slovak, M. L.; McConnell, T. S.; Head, D. R.; Weick, J.; Grever, M. R.; Appelbaum, F. R.; Willman, C. L. Frequency and clinical significance of the expression of the multidrug resistance proteins MDR1/P-glycoprotein, MRP1, and LRP in acute myeloid leukemia: a Southwest Oncology Group Study. Blood, 1999, 94 (3), 1086-1099.

[87] Beck, W. T.; Grogan, T. M.; Willman, C. L.; Cordon-Cardo, C.; Parham, D. M.; Kuttesch, J. F.; Andreeff, M.; Bates, S. E.; Berard, C. W.; Boyett, J. M.; Brophy, N. A.; Broxterman, H. J.; Chan, H. S.; Dalton, W. S.; Dietel, M.; Fojo, A. T.; Gascoyne, R. D.; Head, D.; Houghton, P. J.; Srivastava, D. K.; Lehnert, M.; Leith, C. P.; Paietta, E.; Pavelic, Z. P.; Weinstein, R. Methods to detect Pglycoprotein-associated multidrug resistance in patients' tumors: consensus recommendations. Cancer Res., 1996, 56 (13), 30103020 .

[88] Cianfriglia, M.; Cenciarelli, C.; Barca, S.; Tombesi, M.; Flego, M.; Dupuis, M. L. Monoclonal antibodies as a tool for structurefunction studies of the MDR1-P-glycoprotein. Curr. Protein Pept. Sci., 2002, 3 (5), 513-530.

[89] Goemans, B. F.; Zwaan, C. M.; Vijverberg, S. J.; Loonen, A. H.; Creutzig, U.; Hahlen, K.; Reinhardt, D.; Gibson, B. E.; Cloos, J.; Kaspers, G. J. Large interindividual differences in cellular sensitivity to calicheamicin may influence gemtuzumab ozogamicin response in acute myeloid leukemia. Leukemia, 2008, 22 (12), 2284-2285.

[90] Younes, A.; Bartlett, N. L.; Leonard, J. P.; Kennedy, D. A.; Lynch, C. M.; Sievers, E. L.; Forero-Torres, A. Brentuximab vedotin (SGN-35) for relapsed CD30-positive lymphomas. N. Engl. J. Med., 2010, 363 (19), 1812-1821.

[91] Sutherland, M. S.; Sanderson, R. J.; Gordon, K. A.; Andreyka, J.; Cerveny, C. G.; Yu, C.; Lewis, T. S.; Meyer, D. L.; Zabinski, R. F.; Doronina, S. O.; Senter, P. D.; Law, C. L.; Wahl, A. F. Lysosomal trafficking and cysteine protease metabolism confer target-specific cytotoxicity by peptide-linked anti-CD30-auristatin conjugates. $J$. Biol. Chem., 2006, 281 (15), 10540-10547.

[92] Adams, G. P.; Schier, R. Generating improved single-chain Fv molecules for tumor targeting. J. Immunol. Methods, 1999, 231 (12), 249-260 